\title{
Carnets
}

Revue électronique d'études françaises de l'APEF

Deuxième série - 8 | 2016

Du Français en cause aux causes du français

\section{L'évolution de la didactique des langues impulsée par le CECRL}

Ses traces dans les critères d'évaluation du DELF et dans les manuels de FLE en enseignement secondaire en Andalousie

Nathalie Boiget

\section{(2) OpenEdition}

Journals

Édition électronique

URL : http://journals.openedition.org/carnets/1905

DOI : $10.4000 /$ carnets. 1905

ISSN : 1646-7698

Éditeur

APEF

Référence électronique

Nathalie Boiget, "L'évolution de la didactique des langues impulsée par le CECRL », Carnets [En ligne], Deuxième série - 8 | 2016, mis en ligne le 30 novembre 2016, consulté le 10 décembre 2020. URL:

http://journals.openedition.org/carnets/1905; DOI : https://doi.org/10.4000/carnets.1905

Ce document a été généré automatiquement le 10 décembre 2020.

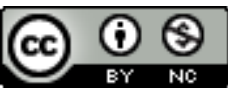

Carnets est mis à disposition selon les termes de la licence Creative Commons - Atribution - Pas

d'utilisation commerciale 4.0 International. 


\title{
L'évolution de la didactique des langues impulsée par le CECRL
}

Ses traces dans les critères d'évaluation du DELF et dans les manuels de FLE en enseignement secondaire en Andalousie

\author{
Nathalie Boiget
}

\section{Introduction}

1 Notre objectif est de démontrer que les innovations pédagogiques en Andalousie (Espagne) dans l'enseignement secondaire ont été clairement influencées par la publication du CECRL en 2001. Pour cela, nous allons commencer notre étude par un état des lieux des méthodologies utilisées en Europe au moment où est impulsée la réflexion du Conseil de l'Europe afin de la contextualiser, puis nous réaliseront une analyse plus ou moins exhaustive du CECRL. Afin d'éviter de laisser de côté des points qui pourraient être importants pour notre sujet, nous avons privilégié la source du document plutôt que de citer des auteurs qui auraient écrit sur ce thème. En effet pour bien comprendre sa portée, il s'avère nécessaire de bien connaitre son contexte de production et son esprit. Pour cela nous nous attarderont plus particulièrement sur l'approche actionnelle qu'il préconise dans tout acte d'apprentissage et d'enseignement d'une langue ainsi que sur les influences des théories énonciatives et discursives qui ont pu accompagner la réflexion des praticiens ayant collaboré à sa rédaction. C'est alors que nous expliquerons à l'aide de l'exemple d'un examen de certification en langue française, le DELF, comment les niveaux communs de référence permettent aux praticiens d'élaborer leur propre réflexion en la basant sur des fondements ou critères communs. Enfin nous nous attacherons à montrer les applications du CECRL dans les manuels de FLE en Andalousie dans les années qui suivirent sa publication. 


\section{Partie 1. L'état des lieux de la didactique des langues en Europe dans les années 70 et 80}

2 En ce qui concerne l'enseignement scolaire des langues étrangères dans un pays donné, nous ne pouvons pas parler d'une succession chronologique de méthodologies, mais nous constatons plutôt des chevauchements, accompagnés de retour en arrière ou encore d'adaptations. D'autre part, même si les sources d'informations sont abondantes (ouvrages de didactique, rapports, instructions officielles...etc.), il reste compliqué d'en déduire les pratiques réelles dans la salle de classe. Nous devrons donc être prudents dans l'interprétation de nos données (Réseau, 2001 : 79).

3 Skinner est considéré comme père du béhaviorisme, courant auquel Bailly, comme la plupart des auteurs, donne une importance très marquante : «[le] courant behavioriste, (...) marquera fortement les conceptions de l'enseignement des langues pendant un demisiècle (...)» (Bailly, $1997: 33$ ). Cette méthodologie, née pour former massivement et rapidement les officiers américains pendant la deuxième guerre mondiale, qui se basait sur l'utilisation intensive de deux machines parlantes (le magnétophone et le phonographe) a eu une grande influence sur la didactique des langues étrangères.

4 La méthode audio-orale est considérée comme l'application du behaviorisme. Elle défend que le langage ne peut être acquis qu'en le pratiquant : la forme orale est prédominante (écouter, puis parler, ensuite lire et enfin écrire), priorité aux situations réelles autant que possible (utilisation des dialogues), l'apprentissage d'une langue est un processus mécanique de formation d'automatismes basé sur la répétition, l'imitation et la vérification immédiate des productions par l'élève (autocorrection), et, enfin la réflexion grammaticale est bannie car l'apprentissage de la grammaire se fait par la mise en place de mécanismes (Réseau, $2001: 81$ ).

5 La méthode audio-visuelle qui suit n'est pas simplement une MAO à laquelle on a rajouté l'image bien qu'elle soit également basée sur le behaviorisme. En effet, l'introduction de l'image au support audio modifie radicalement le processus d'apprentissage du fait de l'effet globalisant sur la communication. Au niveau de la description de la langue, la MAV la plus représentative en France, la SGAV (structuro-globale audio-visuelle), se basait sur l'élaboration d'un corpus de mots les plus fréquents 'le français fondamental' (1959). Selon Cureau, elle est facilement décrite par les quatre mots qui la composent :

- « audio » : priorité à la langue parlée intégrée dans une civilisation et à l'aide du corpus du 'français fondamental'.

- « visuelle» : en situation.

- «structuro » : la langue est considérée sous sa forme structurée sur le plan phonologique et grammatical.

- «global » : méthodologie basée sur une conception globale de la perception (Cureau, 1968).

6 Ces méthodologies ont été attaquées pour l'ambiguïté de l'image qui laisse planer le doute quant à l'interprétation du message, pour représenter une conception excessivement behavioriste de l'apprentissage qui court-circuite la phase analytique de l'apprentissage faite pas l'élève (réflexion sur la langue) et enfin à cause de leurs lacunes dans le domaine de la communication du fait de leur éloignement par rapport à la réalité de la langue et l'authenticité des supports (Réseau, $2001: 89$ ). 
7 Cependant, sans vouloir nier les éléments sur lesquels se sont basées ces critiques, de nombreux auteurs ne partagent pas ces attaques et prétendent que «la méthodologie SGAV a été une entreprise foncièrement révolutionnaire mais trop souvent caricaturée par ceux qui ne l'ont pas pratiquée » (Réseau,2001 : 91), qui cite à ce propos Cureau : « (... ) l'enseignement audio-visuel est un enseignement de communication et d'expression : en aucun cas il ne saurait être un enseignement mécanique (...) »(Cureau,1972:368).

8 Finalement, nous devons reconnaître que la méthodologie SGAV portait en l'état de germe la plupart de concepts qui naîtraient dans les décennies suivantes: approche communicative et cognitive, authenticité, centration sur l'apprenant et développement de l'autonomie de celui-ci (Réseau, $2001: 89$ ). C'est dans ce contexte que commenceront les travaux du Conseil de l'Europe que nous allons aborder dans la partie suivante.

\section{Partie 2. Origine, objectifs et utilisations du CECRL}

9 Le CECRL fut publié en 2001 par le Conseil de l'Europe comme l'aboutissement d'un processus activement mené depuis les années 70 grâce à la collaboration de nombreux membres de la profession enseignante à travers l'Europe. Depuis cette date, le Conseil de l'Europe a joué le rôle de propulseur et catalyseur de la réflexion et des expériences de praticiens des pays européens avec comme objectif de faire avancer l'Europe dans le domaine de l'enseignement des langues. Après bien des aléas, les deux principales réalisations du Conseil de l'Europe ont été 'The Threshold Level' pour l'enseignement de l'anglais comme langue étrangère en contexte européen en 1975, duquel dérivera 'le Niveau seuil' en 1976 pour le français, et enfin le CECRL (Suso López, 2006 : 16). Nous allons axer notre étude sur le CECRL que nous considérons comme le résultat actuel et tangible des politiques européennes d'enseignement des langues menées auparavant, sans pour autant perdre de vue qu'il est bel et bien l'aboutissement de 30 ans de collaboration entre les praticiens.

10 Il propose d'améliorer l'unité entre les membres de la Communauté Européenne, favoriser la mobilité, la compréhension réciproque et la coopération en Europe ainsi qu'éliminer les préjugés et la discrimination à travers une harmonisation de l'enseignement des langues vivantes. Pour cela, il est propulseur de la réflexion et de la coopération entre les praticiens européens et leur fournit une base commune à la description d'objectifs, de contenus et de méthodes. Cependant, il soulève des questions, sans en apporter de réponse et se dit non prescriptif (Conseil de l'Europe, 2001 : pp. 4-10).

11 Par ce souci d'harmonisation à travers une réflexion de chacun des praticiens basée sur des critères communs, il vise tout d'abord à élaborer des programmes adaptés au contexte de chacun qui prennent en compte les connaissances préliminaires. D'autre part, il organise une certification en langue basée sur des examens qui auront des contenus et des critères d'appréciation s'appuyant sur des résultats positifs plutôt que sur des insuffisances. Et enfin, il vise à mettre en place un apprentissage autodirigé par lequel l'apprenant devient acteur de son apprentissage lorsqu'il est capable de définir lui-même ses besoins pour continuer à progresser (Conseil de l'Europe, 2001 : 12). 


\section{Partie 3. L'approche actionnelle du CECRL}

12 Pour le CECRL, la perspective de l'apprentissage et de l'enseignement des langues vivantes sera de type actionnel dans le sens où «l'usager et l'apprenant d'une langue » [sont considérés] « comme des acteurs sociaux ayant à accomplir des tâches (qui ne sont pas seulement langagières) dans des circonstances et un environnement donnés, à l'intérieur d'un domaine donné. » (Conseil de l'Europe, 2001: 15). Nous remarquons que les auteurs du CECRL auraient pu parler 'd'hypothèse actionnelle quant à l'apprentissage' au lieu de 'perspective actionnelle' de l'apprentissage et de l'enseignement, une manière de ne pas «prétendre ouvertement (ou explicitement) bâtir une théorie de l'acquisition des langues ", mais cependant le faire (Suso López, $2006: 361$ ).

Selon la définition du CECRL des caractéristiques de tout acte d'apprentissage ou d'enseignement (approche actionnelle), nous sommes amenés à inclure dans notre réflexion sur nos programmes chacun des points suivants avec un poids variable selon nos objectifs: compétences individuelles, compétences langagières à communiquer, activités langagières, domaines, tâches, stratégies et textes (Conseil de l'Europe, 2001 : 15). Nous allons maintenant nous attacher à décrire chacune de ces dimensions, lesquelles sont fondamentales pour bien comprendre les recommandations du CECRL et ainsi situer les champs de réflexion pour les praticiens, et finalement comprendre les innovations pédagogiques qui suivirent sa publication.

\section{Le CECRL compte quatre compétences générales individuelles}

- Les savoirs ou connaissances déclaratives sont les connaissances résultantes de l'expérience sociale (savoirs empiriques) ou de l'apprentissage formel (savoirs académiques).

- Les habiletés et savoir-faire relèvent de la maîtrise procédurale. Pour mieux les comprendre, il est possible de les comparer au processus d'apprentissage de la conduite automobile. La pratique du permis de conduire fait partie des habiletés et savoir-faire.

- Les savoir-être se constituent des dispositions individuelles, des traits de personnalité ou des attitudes. Ils peuvent être modifiés et faire partie des objectifs d'enseignement également. Les savoir-être sont liés à la culture de chacun et représentent un point sensible lors de l'apprentissage d'une langue.

- Les savoir-apprendre mobilisent quant à eux à la fois les savoirs, savoirs-être, et savoirfaire, tout en s'appuyant sur d'autres compétences comme avoir une prédisposition ou non à connaître l'autre, le sens de l'initiative, savoir rechercher l'information, etc. (Conseil de l'Europe, $2001: 16$ ).

15 La compétence à communiquer langagière est composée de la compétence linguistique, la compétence sociolinguistique et la compétence pragmatique, chacune d'entre elles constituées de savoir, savoir-faire y savoir-être. (Conseil de l'Europe, 2001 : pp. 86-101).

16 Le CECRL définit les compétences linguistiques de la même façon que la plupart des praticiens, pour cette raison, nous n'allons pas insister sur le sujet. La grille du CECRL intitulée « Étendue linguistique générale » (Conseil de l'Europe, 2001 : 87) nous donne un résumé des descripteurs de cette compétence. 
17 La compétence sociolinguistique se reflète dans les connaissances et habiletés nécessaires pour utiliser avec succès la langue dans sa dimension sociale (marqueurs des relations sociales, règles de politesse, expressions de la sagesse populaire, différences de registre, dialecte et accent).

La compétence pragmatique quant à elle «recouvre l'utilisation fonctionnelle des ressources de la langue (réalisation de fonctions langagières, d'actes de parole) en s'appuyant sur des scénarios ou des scripts d'échanges interactionnels » (Conseil de l'Europe, $2001: 18$, pp.96-101). Nous pouvons y inclure directement la maîtrise du discours, de sa cohérence et cohésion, la reconnaissance des types et genre de texte et les effets de la subjectivité du langage comme l'ironie.

Quant aux activités langagières, dans la perspective actionnelle de l'apprentissage et de l'enseignement des langues, nous nous plaçons dans la réalisation de tâches langagières, de ce fait, elles se composent d'activités de type "réception» (écoute, lecture), "production" (expression orale ou écrite), interaction écrite ou orale et médiation (traduction, interprétation, résumé ou compte rendu traitant un « déjà dit » (Conseil de l'Europe, $2001: 18$ ).

Pour l'enseignement et l'apprentissage des langues, le CECRL considère que « tout acte de parole s'inscrit dans le contexte d'une situation donnée, dans le cadre de l'un des domaines (sphères d'activité ou centres d'intérêt) de la vie sociale. » (Conseil de l'Europe, 2001: pp. 41-43) et il définit quatre domaines principaux: le domaine personnel, le domaine public, le domaine professionnel et le domaine éducationnel.

21 L'approche est essentiellement actionnelle et se trouve ainsi centrée sur la relation entre l'apprenant, la tâche qui lui est présentée et les stratégies qu'il va utiliser pour la réaliser. Dans cette perspective, tâche devrait être « authentique » ou encore « réelle » (réaliser un montage ou une recette de cuisine, préparer une réunion ou un voyage...etc.) et basée sur des textes authentiques, mais souvent elle sera pédagogique, c'est-à-dire fictive, adaptée aux possibilités souvent limitées de la classe (jeux de rôle, organisation d'un pseudo événement imaginé, etc.). Cependant dans les deux cas, la tâche sera toujours communicative du fait qu'elle oblige les apprenants à en comprendre, négocier et exprimer le sens afin d'atteindre le but communicatif (Conseil de l'Europe, $2001: 19$ ).

\section{Partie 4. Le CECRL, l'élaboration des curriculums en Europe et son lien avec les théories énonciatives et discursives}

En ce qui concerne son lien avec l'élaboration des curriculums en Europe, il convient de rappeler que le CECRL se veut non dogmatique. Le Conseil de l'Europe ne recommande pas une méthodologie concrète et il considère que « les méthodes à mettre en œuvre pour l'apprentissage, l'enseignement et la recherche sont celles que l'on considère comme les plus efficaces pour atteindre les objectifs convenus en fonction des apprenants concernés dans leur environnement social » (Conseil de l'Europe, 2001: 110). Bien que le Conseil de l'Europe ait soutenu des méthodologies fondées sur les besoins communicatifs des apprenants pendant de longues années, le CECRL ne fera que citer les plus utilisées dans le but de favoriser l'échange d'information et la réflexion de chaque utilisateur. Il n'en prescrit aucune en particulier. Cependant la description que nous venons de faire de l'approche actionnelle nous met directement sur le chemin de l'approche communicative 
revue et corrigée avec l'inclusion de l'apprentissage cognitif (réflexion sur la langue et l'apprentissage, auto-évaluation, construction des savoirs). D'ailleurs lorsque Javier Suso cite le CECRL «En ce qui concerne la capacité à apprendre, on attendra/exigera des apprenants qu'ils développent leurs capacités à apprendre et leurs aptitudes à la découverte lorsqu'ils acceptent la responsabilité de leur propre apprentissage (...)» (Conseil de l'Europe, 2001 : 114), il souligne de suite qu'« il va sans dire que cette option n'est qu'une application de plus de la théorie constructiviste, qui continue de marquer le grand sentier par où avance l'apprentissage (des langues ou des autres matières) en ce début du XXI ${ }^{e}$ siècle. » (Suso López, $2006: 364$ ).

Les rapprochements possibles avec les théories énonciatives et discursives sont multiples. La question de la situation de communication et de la construction du sens pose la base des recommandations du CECRL avec la définition qu'il donne des domaines (citée cidessus) : « Tout acte de parole s'inscrit dans le contexte d'une situation donnée, dans le cadre de l'un des domaines (sphères d'activité ou centres d'intérêt de la vie sociale.)", (Conseil de l'Europe, 2001 : pp. 41-43). Cette approche rejoint la définition de Benveniste de l'énonciation: "mise en fonctionnement de la langue par un acte individuel d'utilisation » (Benveniste, 1974).

Quant à sa description des compétences à communiquer langagières qui serviront de base aux objectifs, contenus et évaluation, le CECRL va décrire les compétences linguistiques (lexicale, grammaticale, sémantique...etc.) en adoptant la démarche de la plupart des linguistes actuels: «La plupart des linguistes descriptifs se contentent désormais de codifier la pratique, mettant en rapport forme et sens et utilisant une terminologie qui ne s'éloigne de la pratique traditionnelle que (...) » (Conseil de l'Europe, $2001:$ 87).

D'autre part, la compétence sociale peut se rapprocher de la théorie des actes de langages dont (Austin, 1970) est le père. Il a pris le contre-pied des approches logiques du langage (structuralisme de Saussure, puis Chomsky) et s'est intéressé aux nombreux énoncés qui tels les questions ou les ordres, échappent à la problématique du vrai et du faux car ils ont comme finalité réelle de provoquer une action chez l'interlocuteur qui n'est pas forcément celle qui est décrite par les mots.

Enfin, les quatre maximes conversationnelles (Grice, 1975) qui prennent appui sur le principe de coopération sont directement citées dans la description de la compétence discursive, qui forme partie de la compétence pragmatique, comme «(...) la capacité de gérer et de structurer le discours en terme, entre autres, de principe coopératif (maximes conversationnelles de Grice, 1975) »(Conseil de l'Europe, $2001: 96$ ).

Ces quelques exemples nous montrent combien la réflexion sur le CECRL a été influencée par les théories énonciatives et discursives des années qui l'ont précédé. Maintenant nous allons examiner des applications concrètes dans l'enseignement du FLE survenues suite à cette évolution de la didactique des langues.

\section{Partie 5. Des exemples d'applications du CECRL : un examen de certification (le DELF) et les manuels de FLE en enseignement secondaire en Andalousie} de survie, B1 : niveau seuil, B2 : avancé ou indépendant, $\mathrm{C} 1$ : autonome, $\mathrm{C} 2:$ maîtrise) 
fournissent une grille conceptuelle, toujours hors contexte, qui sera utile aux utilisateurs pour décrire leur propre système. Chaque praticien est invité à réfléchir sur l'élaboration de ses programmes et son évaluation en fonction du contexte dans lequel il travaille (Conseil de l'Europe, 2001 : pp. 24-28).

Les trois tableaux principaux: "Tableau 1-Niveaux communs de référence de compétence-Échelle globale », «Tableau 2-Grille pour l'autoévaluation » et «Tableau 3Aspects qualitatifs de l'utilisation de la langue parlée " (Conseil de l'Europe, 2001: pp. 25-28), qui définissent les niveaux de référence de manière beaucoup plus détaillée dans le chapitre III du CECR renvoient toujours aux trois métacatégories suivantes :

- Les activités communicatives qui sont liées à des descripteurs de capacités à faire présents dans la réception, l'interaction et la production.

- Les stratégies qui relèvent également de descripteurs de capacité à faire, cependant attachée à une stratégie, non à une activité communicative. Elles sont la charnière entre les compétences de l'apprenant et ce qu'il peut en faire (les activités communicatives). Elles se développent selon les principes suivants : planification de l'action, évaluation des ressources (compétences), compensation des déficiences au cours de l'exécution, contrôle des résultats et ajustement (rétro alimentation).

- Et les compétences communicatives langagières qui se relient à des descripteurs étalonnés par niveau (de A1 à C2) pour les compétences linguistiques, communicatives et sociolinguistiques.

Á titre d'exemple d'application et de flexibilité des descripteurs, nous citerons le DELF, examen de certification en langue française. Il a adopté les niveaux communs de référence proposés par le CECRL ainsi que son système d'échelles qu'il a su adapté aux nécessités des candidats : DELF tout public, DELF Prim, DELF Scolaire, DELF Junior, DELF Pro et DALF pour les niveaux $\mathrm{C} 1$ et $\mathrm{C} 2$. Plus concrètement, nous allons maintenant donner un exemple de cette affirmation en comparant un des descripteurs des niveaux de compétence du CECRL du «Tableau 2-Grille pour l'autoévaluation » (...) « Prendre part à une conversation, niveau B2 " (Conseil de l'Europe, 2001: pp. 26-27) avec celui qui lui correspond pour l'évaluation des candidats au DELF niveau B2 lors de la production orale, " 2 ième partie, exercice en interaction: débat", à l'aide du document réservé aux correcteurs et sa grille d'évaluation de la production orale, située en page $19 \mathrm{du}$ document (CIEP. «Diplôme d'étude en langue française DELF B2» [on-line], p. 19 [disponible le 29/08/2016] <URL: http://www.ciep.fr/delf-tout-public/exemples-dessujets>).

\begin{tabular}{|l|l|l|}
\hline \multicolumn{2}{|l|}{ Comparaison des descripteurs de CECRL avec ceux du DELF (interaction orale) } \\
\hline $\begin{array}{l}\text { Tableau 2-Grille pour l'autoévaluation (Conseil de l'Europe, 2001: } \\
\text { Ex. 26-27). }\end{array}$ & $\begin{array}{lr}\text { dxamen du DELF, } \\
\text { document réservé aux } \\
\text { correcteurs. }\end{array}$ \\
\hline
\end{tabular}


«Je peux communiquer avec un degré de spontanéité et d'aisance qui rend possible une interaction normale avec un locuteur natif. Je peux participer activement à une conversation dans des situations familières, présenter et défendre mes opinions. »

«Peut confirmer et
nuancer ses idées et ses
opinions, apporter des
précisons.
Peut réagir aux arguments
et déclarations d'autrui
pour défendre sa
position».

31 À présent, à l'aide de l'étude résumée dans le tableau ci-dessous, nous allons rechercher les marques d'application du CECRL dans des manuels de FLE en enseignement secondaire en Andalousie qui nous semblent représentatifs.

\begin{tabular}{|c|c|c|c|c|c|c|}
\hline $\begin{array}{l}\text { Les critères de l'approche } \\
\text { actionnelle: leurs traces dans } \\
\text { les manuels de FLE en } \\
\text { éducation secondaires en } \\
\text { Andalousie }\end{array}$ & $\begin{array}{l}\text { Tous à } \\
\text { bord } 4 \\
(2007)\end{array}$ & $\begin{array}{l}\text { Essentiel } \\
3 \\
(2007)\end{array}$ & $\begin{array}{l}\text { Ça } \\
\text { marche } \\
3(2008)\end{array}$ & $\begin{array}{l}\text { Atelier } \\
4 \\
(2010)\end{array}$ & $\begin{array}{l}\text { @robase } \\
2 \\
(2014)\end{array}$ & $\begin{array}{l}\text { Parachute } \\
3 \\
(2015)\end{array}$ \\
\hline $\begin{array}{l}\text { Mention des niveaux communs de } \\
\text { référence du CECRL ou du DELF }\end{array}$ & $\mathrm{x}$ & $\mathrm{x}$ & $\mathrm{X}$ & $\mathrm{X}$ & $\mathrm{X}$ & $\mathrm{x}$ \\
\hline $\begin{array}{l}\text { La tâche visant la communication } \\
\text { langagière }\end{array}$ & & & & & & \\
\hline Tâche authentique (réelle) & & $\mathrm{x}$ & $\mathrm{X}$ & $\mathrm{X}$ & $\mathrm{x}$ & $\mathrm{x}$ \\
\hline $\begin{array}{l}\text { Tâche pédagogique proche d'une } \\
\text { tâche authentique }\end{array}$ & $\mathrm{x}$ & $\mathrm{x}$ & $\mathrm{X}$ & $\mathrm{X}$ & $\mathrm{X}$ & $\mathrm{x}$ \\
\hline Tâche purement pédagogique & $\mathrm{x}$ & $\mathrm{x}$ & $\mathrm{x}$ & $x$ & $\mathrm{x}$ & \\
\hline Les savoirs développés & & & & & & \\
\hline $\begin{array}{l}\text { Les savoirs (connaissances } \\
\text { déclaratives) }\end{array}$ & $\mathrm{x}$ & $\mathrm{x}$ & $\mathrm{X}$ & $\mathrm{X}$ & $\mathrm{X}$ & $\mathrm{X}$ \\
\hline $\begin{array}{l}\text { Les habiletés et savoir-faire } \\
\text { (stratégies pour réaliser la tâche) }\end{array}$ & $\mathrm{x}$ & $\mathrm{x}$ & & $\mathrm{X}$ & $\mathrm{X}$ & $\mathrm{x}$ \\
\hline Les savoir-être & & $\mathrm{x}$ & $\mathrm{X}$ & $\mathrm{x}$ & & $\mathrm{x}$ \\
\hline Les savoir-apprendre & & $\mathrm{x}$ & $\mathrm{X}$ & $\mathrm{x}$ & & $\mathrm{x}$ \\
\hline $\begin{array}{l}\text { Les principales compétences } \\
\text { développées }\end{array}$ & & & & & & \\
\hline $\begin{array}{l}\text { Compétences } \\
\text { linguistiques (lexical, } \\
\text { grammatical) }\end{array}$ & $\mathrm{X}$ & $\mathrm{X}$ & $\mathrm{X}$ & $\mathrm{X}$ & $\mathrm{X}$ & $\mathrm{X}$ \\
\hline
\end{tabular}




\begin{tabular}{|c|c|c|c|c|c|c|}
\hline Compétence phonologique & $\mathrm{X}$ & $\mathrm{x}$ & $\mathrm{x}$ & $\mathrm{X}$ & $\mathrm{x}$ & $\mathrm{x}$ \\
\hline Compétence sociologique & $\mathrm{X}$ & $\mathrm{x}$ & $\mathrm{X}$ & $\mathrm{X}$ & $\mathrm{x}$ & $\mathrm{x}$ \\
\hline Compétence pragmatique & $\mathrm{x}$ & $\mathrm{x}$ & $\mathrm{X}$ & $\mathrm{X}$ & $\mathrm{x}$ & $\mathrm{x}$ \\
\hline $\begin{array}{l}\text { Le texte et les activités } \\
\text { langagières }\end{array}$ & & & & & & \\
\hline Présence de textes authentiques & $\mathrm{x}$ & $\mathrm{x}$ & $\mathrm{x}$ & $\mathrm{x}$ & $\mathrm{x}$ & $\mathrm{x}$ \\
\hline $\begin{array}{l}\text { Écoute, production orale, lecture } \\
\text { et production écrite }\end{array}$ & $\mathrm{X}$ & $\mathrm{x}$ & $\mathrm{x}$ & $\mathrm{X}$ & $\mathrm{x}$ & $\mathrm{X}$ \\
\hline Interaction écrite et orale & $\mathrm{X}$ & $\mathrm{x}$ & $\mathrm{x}$ & $\mathrm{X}$ & $\mathrm{x}$ & $\mathrm{x}$ \\
\hline $\begin{array}{l}\text { Médiation (reformulation ou } \\
\text { traduction) }\end{array}$ & $x$ & $\mathrm{X}$ & $\mathrm{X}$ & X & $\mathrm{X}$ & $\mathrm{X}$ \\
\hline $\begin{array}{l}\text { Exercices de vocabulaire et } \\
\text { grammaire }\end{array}$ & $\mathrm{X}$ & Peu & Peu & $\mathrm{X}$ & Peu & \\
\hline Règle de grammaire puis activité & $\mathrm{X}$ & & & & & \\
\hline $\begin{array}{l}\text { Activité, puis déduction de la } \\
\text { grammaire }\end{array}$ & & $\mathrm{X}$ & $\mathrm{X}$ & $\mathrm{X}$ & $\mathrm{X}$ & $\mathrm{X}$ \\
\hline
\end{tabular}

\section{Interprétation du tableau ci-dessus}

Pour réaliser l'étude, nous avons choisi des livres parus au moins 6 ans après la publication du CECRL car en Espagne la première loi d'éducation (la LOE) qui a pu éventuellement imposer les critères de ce dernier est entrée en vigueur à partir de 2006, sachant qu'il faut prendre également en compte le délai nécessaire pour les décrets d'application de celle-ci. L'étude s'est basée sur l'approche retenue par le CECRL dans son chapitre 2, l'approche actionnelle (Conseil de l'Europe, 2001: pp.15-22). Ainsi dans le tableau ci-dessus, nous avons sélectionné des critères d'application propre à cette perspective.

De plus, nous tenons à préciser que nous entendons par compétence sociologique la connaissance d'autres cultures incluant marqueurs des relations sociales, politesse, différences de registre et la sagesse populaire. Nous parlons de présence de textes authentiques car aucun des manuels que nous connaissons n'est construit uniquement avec ce genre de textes. Le « $\mathrm{X}$ » signifie que ce critère est utilisé à chaque leçon dans le livre, l'espace blanc marque son absence complète, «peu » est synonyme d'une présence très réduite.

Nos conclusions sur l'interprétation du tableau ci-dessus sont les suivantes :

Après 2006, tous les manuels font toujours référence au CECRL, garantissant qu'ils répondent à ses recommandations. 

extraits d'un des manuels utilisés pour l'étude, «Parachute 3 »:

- Compétence sociologique : "Atelier d'écriture. Savoir remercier » (Parachute 3, 2015 : 61).

- Compétence pragmatique: lecture théâtralisée d'un conte qui les amènera à l'aide de conseils à jouer le conte en groupe (Parachute 3, 2015 : 74)

- Tâche authentique dans laquelle ils doivent répondre à un sondage puis en réaliser un autre dans la classe et finalement en rédiger les résultats (Parachute 3, $2015: 39$ ).

- Habiletés et des savoir-faire (stratégies pour réaliser la tâche) : Activité « Trucs et astuces... (...) Pour parler en public » (Parachute 3, $2015: 39$ ).

- Savoir-être page 57 : une activité portant sur l'amitié (Parachute 3, 2015 : 57).

- Savoir apprendre : auto-évaluation avec des exercices types « DELF » (Parachute 3, 2015 : 32) et réflexion sur la langue « J'observe et j'analyse [...] Qu'est-ce que tu utilises pour construire le futur simple? » (Parachute 3, $2015: 37$ ).

\section{Conclusion générale}

41 Notre étude de l'application pratique du CECRL se limite à un échantillon de manuels de FLE en éducation secondaire en Andalousie qu'il serait peut-être imprudent de généraliser à l'Europe entière ou même à l'Espagne dans son ensemble : nous proposons ici une ouverture sur une autre étude qui consisterait en analyser davantage de manuels 
de FLE d'éducation secondaire en Espagne, et éventuellement, étudier leur évolution dans l'avenir pour confirmer nos résultats. Cependant, pour l'instant le constat provisoire serait le suivant: après 2001, nous observons une claire influence du CECRL sur les manuels de FLE en éducation secondaire en Andalousie qui nous place sur le chemin d'une méthodologie communicative revue et corrigée avec l'inclusion de l'apprentissage cognitif (réflexion sur la langue et l'apprentissage, auto-évaluation, construction des savoirs), basée sur une approche actionnelle de l'enseignement apprentissage.

Les praticiens ont inscrit les descripteurs des six niveaux de compétence dans leurs manuels de FLE d'éducation secondaire en Andalousie ainsi que dans l'examen certification de FLE de référence du ministère de l'éducation français (le DELF), ce qui démontre qu'ils les perçoivent comme un outil de calibrage qui permet de travailler avec des normes stables et reconnues dans toute l'Europe, facilitant la communication, la mobilité et les échanges. Finalement, ces niveaux donnent un moyen de suivre le progrès des apprenants au fur et à mesure qu'ils construisent leur compétence à travers les paramètres des schémas descriptifs.

Enfin la définition de l'acte de communication que nous propose Sophie Moirand comme un dispositif dans lequel interviennent : une situation de communication (cadre physique et mental), des interlocuteurs possédant une identité et reliés par un contrat de communication, un mode d'organisation du discours (qui dépendent de la finalité communicative de l'émetteur), la langue (forme et sens) et le texte (résultat final de l'acte de communication), pourrait être facilement reliée à la description de l'approche actionnelle du CECR (Moirand, 1990).

\section{BIBLIOGRAPHIE}

ADIDA, A., MORENO, E., courtier, S. (2015). Parachute 3. Madrid : Santillana.

AUSTIN, J. L. (1970). Quand dire, c'est faire. Paris : Seuil.

BENVENISTE, E. (1966). Problèmes de linguistique générale. Paris : Gallimard.

CALlejA, I., SEgARRA, M. (2007). Ça marche 3. Madrid: Pearson Educación.

CHомsкY, N. (1959). Structures syntaxiques. Paris : Seuil.

CIEP. "Diplôme d'étude en langue française DELF B2" [on-line], p. 19 [disponible le 29/08/2016]

<URL : http://www.ciep.fr/delf-tout-public/exemples-des-sujets>.

CONSEIL DE L'EUROPE (2001). « Cadre européen commun de référence pour les langues. Apprendre, enseigner, évaluer. » [on-line]. Paris : Didier [disponible le 30/08/2016] <URL : http://

www.coe.int/lang-CECR>.

CUlioli, A. (1990). Pour une linguistique de l'énonciation. Opérations et représentations. Tome 1, Editions Ophrys.

CUREAU, J. (1968) « La méthode audio-visuelle structuro-globale SaintCloud-Zagreb », Les Langues

Modernes, $n^{\circ} 4$, p. 461-462. 
GALISSON, R. (1990). « Où va la didactique du français langue étrangère ? », Études de Linguistique Appliquée, $n^{\circ}$ 79, juillet-septembre, pp. 9-34. Paris : Didier-Érudition.

GALLON F., HIMBER C., RASTELlo C. (2010). Atelier 4, Paris : Hachette langue étrangère, S.M. Français. GERMAIN, C. (1991). L'approche communicative en didactique des langues. Anjou, (Québec, Canada) : Centre éducatif et Culturel inc. $104 \mathrm{p}$.

MAINGUENEAU, D. (1981). Approche de l'énonciation en linguistique française. Paris : Hachette.

MOIRAND, S. (1982). 4e édition 1992. Enseigner à communiquer en langue étrangère. Paris : Hachette.

MOIRAND, S., (1990). Une grammaire des textes et des dialogues. Paris : Hachette.

MORENO, E., COUTURIER, S. (2007). Essentiel 3. Madrid : Santillana.

PUREN, C. (1988). Histoire des méthodologies de l'enseignement des langues. Paris : CLE International, coll. DLE.

RÉZEAU, J. (2001). Médiatisation et médiation pédagogique dans un environnement multimédia. Thèse.

Bordeaux : Université Victor Segalen Bordeaux 2.

SAUSSURE, F. De 1995 (1e éd. 1916). Cours de linguistique général. Paris : Payot, coll. » Grande bibliothèque Payot ».

schmiтt, S. (2011). @robase 2. Varese: Clé international, Oxford Educación.

SKINNER, B. F. (1968) The technology of teaching. New York: Appleton Century Crofts. Trad.

française : La révolution scientifique de l'enseignement. Bruxelles : Dessart.

SPANO, C., MARÉVA, B., BERTINI, J. (2007). Tous à bord 4. Bacelona : Vicens Vives.

SUSO LÓPEZ, J. (2006). De un « Niveau-Seuil » au « Cadre européen commun de référence pour les langues ». Grenade-Barcelone : Université de Grenade.

\section{RÉSUMÉS}

Notre étude a pour but de décrire les innovations concernant la didactique des langues étrangères liées à la publication du CECRL en 2001, plus concrètement celles qui sont visibles dans des manuels de FLE utilisés en éducation secondaire en Andalousie. Elle s'appuie sur une analyse exhaustive du CECRL qui aboutit à une description explicative de l'examen de certification en langue française par excellence, le DELF, en tant qu'application directe de ce dernier, puis à une enquête sur les manuels de FLE en éducation secondaire en Andalousie qui fait ressortir également une grande imbrication. Cette étude démontre également comment dans la pratique il a joué un rôle dans la transformation de la didactique des langues conformément à l'évolution des théories énonciatives et discursives dans les dernières décennies. En effet, c'est grâce à celles-ci et leur influence dans le monde de l'enseignement, que nous sommes passés des grammaires normatives, puis fonctionnelles, génératives ou transformationnelles à une "grammaire " de la communication qui pointe sur l'usage de la langue, qui décrit les faits de langages en fonction des intentions du sujet parlant et des attentes des interlocuteurs, qui s'intéresse enfin aux enjeux pragmatiques et aux pratiques discursives, avec des conséquences fondamentales sur la sélection des contenus des cours de langue étrangère, les instruments, le mode d'organisation de la classe et le corpus.

The purpose of this article is to describe the innovations of foreign language didactics according to The Common European Framework of Reference for Languages (CEFR henceforward), above 
all, all those that are connected with FLE (French as a foreign language) textbooks used in secondary education in Andalusia. It relies on an exhaustive analysis of the CEFR as well as a description of the French certification exam par excellence, Diplôme d'Études en Langue Française (DELF henceforward), as its direct application, and finally an investigation of secondary education FLE textbooks in Andalusia, which ends in a similar imbrication. This study also shows the transformation of foreign language didactics in accordance with the last decade enunciative pragmatics theories evolution. Indeed, the influence in the world of education enables the change from normative grammar, then functional, generative or transformational to communicative grammar. The latter points out the language use, describes speech acts according to the speaker's intentions and interlocutor's expectations, and it is interested in pragmatics issues, with essentials consequences in FLE course contents, instruments, classroom organization and corpus.

\section{INDEX}

Mots-clés : didactique, CECRL (Cadre européen commun pour les langues), approche actionnelle, niveaux communs de référence

Keywords : didactics, CEFR (Common European Framework of Reference for Languages), action approach, common reference levels

\section{AUTEUR}

NATHALIE BOIGET

Université de Cadix

nathalie.boiget[at]uca.es 